\title{
Minireview \\ From bending DNA to diabetes: the curious case of HMGA1
}

\author{
Robert K Semple
}

Address: Metabolic Research Laboratories, Institute of Metabolic Science, University of Cambridge, Addenbrooke's Hospital, Cambridge CB2 0QR, UK. Email: rks16@cam.ac.uk

\begin{abstract}
Although mice lacking the architectural DNA binding factor HMGA1 are diabetic and express very low levels of the insulin receptor, they have increased insulin sensitivity. A study in $B M C$ Biology now suggests that changes in circulating retinol binding protein partly account for this paradox.
\end{abstract}

The inexorable rise of pandemic diabetes mellitus is already leaving a devastating global trail of premature debility and mortality in its wake. Around 90\% of patients with diabetes ('mellitus' will be assumed from here on) have type 2 diabetes (T2DM), which is intimately associated with and driven by increasing levels of obesity. However, despite intensive investigation in humans and model organisms, many aspects of its pathogenesis remain unclear, and there is an imperative need to refine understanding of the disease process in order to develop new and rational therapies.

Difficulty in elucidating the precise pathological basis of T2DM stems in part from its heterogeneity - indeed, the certainty implied by this apparently specific label, applied by default to most patients without collateral diagnostic features of other, better defined types of diabetes, may be illusory. It is true that most patients with T2DM do share certain general characteristics - they are mostly overweight or obese, mostly in middle age or beyond, and mostly have normal or elevated insulin at diagnosis - but extensive clinical observation suggests that the predominant physiological defect(s) commonly differs significantly among them. Such heterogeneity is not surprising a priori intermediary metabolism is regulated in a complex manner by constant metabolic dialog among organs, including brain, liver, fat, pancreas and skeletal muscle. This is necessary to serve the sometimes competing organismal needs of growth, reproduction and day-to-day survival in the face of fluctuating nutritional and environmental inputs, but the number of 'moving parts' in this complex system also renders it susceptible to perturbation in many different ways. Chiefari et al. [1] now report in $B M C$ Biology the latest installment of a fascinating - and sometimes perplexing - line of investigation that has been running for the past 20 years, centered on the DNAbinding protein HMGA1. The new findings not only give intriguing insights into metabolic crosstalk between tissues in vivo, but also illustrate several key themes and challenges in current T2DM research.

\section{HMGA1 in human severe insulin resistance}

The story began more than two decades ago with identification of a boy with high glucose levels despite extremely elevated insulin - that is, severe insulin resistance (IR) [2]. His blood cells showed very low insulin binding and insulin receptor expression, and yet, surprisingly, neither of the alleles of the gene encoding the insulin receptor, INSR, had any mutation [3]. Later independent studies found that the protein HMGA1 was bound to key sites in the promoter of the INSR gene, and provided evidence that HMGA1 is involved in the high levels of INSR expression in insulinresponsive tissues [4]. HMG (high mobility group) proteins are the second most abundant nuclear proteins after histones, and proteins in the HMGA subfamily, although without intrinsic transcriptional activating activity, function as permissive 'architectural' transcription factors, binding AT-rich sequence motifs, distorting DNA and nucleating assembly of complexes of other factors with canonical transactivation motifs [5]. These biochemical and clinical genetic lines of investigation converged in 2004 with the demonstration that the insulin-resistant proband, as well as three further patients with severe IR, had extremely low levels of $H M G A 1$ expression, in all cases as a result of mutations or deletions severely reducing levels of HMGA1 mRNA [6].

This biomedical detective work was of direct importance to these patients with $H M G A 1$ defects, but the question arises as to whether such 'boutique' examples of rare monogenic IR have any relevance to the common forms of IR and T2DM, which, although they show high heritability, are thought likely to be predisposed to by milder defects in several different genes. The most powerful genetic tool used to seek association between common genetic variation and disease is the genome-wide association study, but such studies have explained only a tiny proportion of the heritability of T2DM so far. Two possible interpretations of 
this are (i) that different types of rare genetic variation collectively have a more dominant role in T2DM susceptibility than common genetic variation, and/or (ii) many patients in the non-diabetic control group may be in the long prodromal phase of IR, which precedes pancreatic beta cell decompensation and hyperglycemia and is commonly asymptomatic, attenuating the power of genome-wide association studies to detect genetic differences between the groups. The relatively disappointing yield from population-wide genetics makes study of monogenic forms of IR or diabetes, in which the primary defect is known, and its comparison with common T2DM/IR, in which the primary defect is unclear, particularly valuable, as exemplified by humans with INSR defects [7]. Identification of such human 'experiments of nature' also serves to validate candidate genes for more detailed resequencing studies in wider populations.

\section{HMGA1 in mice and humans}

There are significant practical and ethical limitations to study of patients, however, and only four patients with HMGA1 mutations have been reported in total so far. $\mathrm{Hmga1}^{-/}$mice were therefore generated for more detailed mechanistic study [6]. In keeping with the human phenotype, these animals showed a 90\% reduction in insulin receptor expression and hyperglycemia after glucose challenge. In other respects, however, the phenotypes of mice and humans radically diverged: all affected humans had severe peripheral IR and corresponding hyperinsulinemia, whereas knockout mice were actually hypersensitive to insulin, with increased levels of expression of the insulin-responsive glucose transporter Glut4 in muscle and enhanced whole-body insulinstimulated glucose disposal (Table 1). This is in effect the opposite phenotype to that in humans, and implies major differences in both insulin secretion and peripheral insulin action between the species. The earlier failure of insulin secretion in mice than humans suggests a failure of glucose-stimulated insulin secretion in individual pancreatic $\beta$ cells and/or failure of proliferation of these cells. This may relate to a more important role for the Insr in this process in mice, as suggested by the progressive hyperglycemia of beta-cell-specific Insr knockout mice [8]. The difference in peripheral insulin sensitivity, suggesting the existence of a pathway in mice that can compensate for severe loss of Insr, was left unexplained, however.

\section{HMGA1 and metabolic messengers}

The contribution of the recent paper by Chiefari et al. [1] is to offer a partial explanation for this observation. The new work draws on growing appreciation that insulin-sensitive tissues communicate not only through substrate fluxes, but also by secreting factors that signal specifically to distant tissues. Adipose tissue, in particular, is now seen as a highly dynamic endocrine organ producing many so-called 'adipokines', including leptin, which has a thoroughly validated metabolic role, adiponectin, and many other factors with varying credentials as bona fide signaling molecules. One of the more recent of these is retinol binding protein (RBP), product of the Rbp4 gene in mice. Although mostly produced by the liver, RBP also comes from white adipose tissue, and its expression there is inversely related to Glut4 expression [9]. Furthermore, $R b p 4^{-/}$mice show enhanced insulin sensitivity, and providing RBP either exogenously or by overexpression induces IR [9].

Chiefari et al. [1] hypothesized that altered Rbp4 expression might explain the discordance between reduced Insr

Table 1

Insulin resistance 'subphenotypes' seen in humans and mice with deficiency or mutations of HMGA1 or INSR*

\begin{tabular}{|c|c|c|c|c|c|c|}
\hline \multirow[b]{2}{*}{ Tissue } & \multirow[b]{2}{*}{ Characteristic } & \multicolumn{3}{|c|}{ Human } & \multicolumn{2}{|r|}{ Mouse } \\
\hline & & INSR mutation ${ }^{\dagger}$ & HMGA1 mutation & Common IR/T2DM & Hmga1\%- & Insr loss of function \\
\hline \multirow[t]{4}{*}{ Whole body } & Fasting glucose & $\downarrow$ to $\rightarrow$ to $\uparrow$ & $\downarrow$ to $\rightarrow$ to $\uparrow$ & $\rightarrow$ to $\uparrow$ & $\rightarrow$ & $\downarrow$ to $\rightarrow$ to $\uparrow$ \\
\hline & Fasting insulin & $\uparrow \uparrow \uparrow$ & $\uparrow \uparrow \uparrow$ & $\uparrow$ to $\uparrow$ & $\downarrow$ & $\uparrow \uparrow$ \\
\hline & Insulin sensitivity & $\downarrow \downarrow \downarrow$ & $\downarrow \downarrow \downarrow$ & $\downarrow$ to $\downarrow \downarrow$ & $\uparrow$ & $\downarrow \downarrow \downarrow$ \\
\hline & Glucose tolerance & $\rightarrow$ to $\downarrow$ & $\rightarrow$ to $\downarrow$ & $\rightarrow$ to $\downarrow$ & $\downarrow$ & $\downarrow$ \\
\hline \multirow[t]{4}{*}{ Liver } & Lipid profile & Normal Tg and HDL & $?$ & High Tg, low HDL & $?$ & $?$ \\
\hline & IGFBP1 & $\uparrow$ & $\downarrow$ & $\downarrow$ & $\downarrow$ & $\uparrow \uparrow$ \\
\hline & SHBG & $\uparrow$ & $?$ & $\downarrow$ & $?$ & $?$ \\
\hline & Liver fat & Normal & $?$ & $\uparrow$ & $?$ & $?$ \\
\hline \multirow[t]{3}{*}{ Adipose tissue } & Adiponectin & $\uparrow \uparrow$ & $?$ & $\downarrow$ & $?$ & $\uparrow$ \\
\hline & Leptin & $\downarrow$ & $?$ & $\rightarrow$ to $\uparrow \uparrow$ & $?$ & $\downarrow$ \\
\hline & RBP & $\downarrow$ & $?$ & $\uparrow$ & $\downarrow \downarrow$ & $?$ \\
\hline
\end{tabular}

${ }^{*}$ Tg, triglyceride; HDL, high density lipoprotein cholesterol; IGFBP1, insulin-like growth factor binding protein 1; SHBG, sex hormone binding globulin; RBP, retinol binding protein. ${ }^{\dagger}$ Representative of patients with Rabson Mendenhall syndrome, in which there is approximately $90 \%$ loss of INSR function. ₹No strictly Insr hypomorphic mice with 10\% residual receptor function have been reported, so results are inferred from related models, such as a conditional peripheral Insr knockout and an Insr mosaic knockout. 
(a)

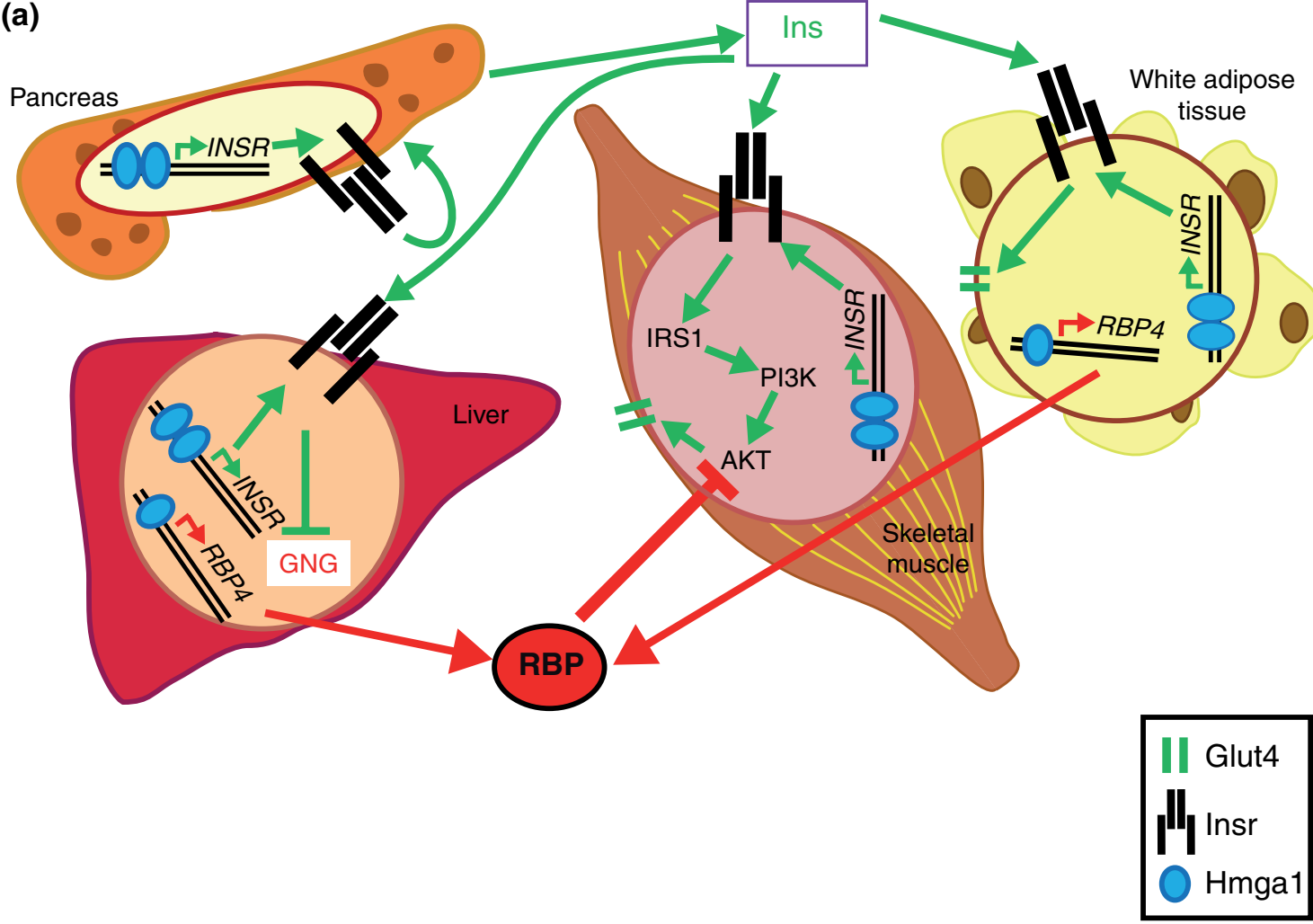

(b)

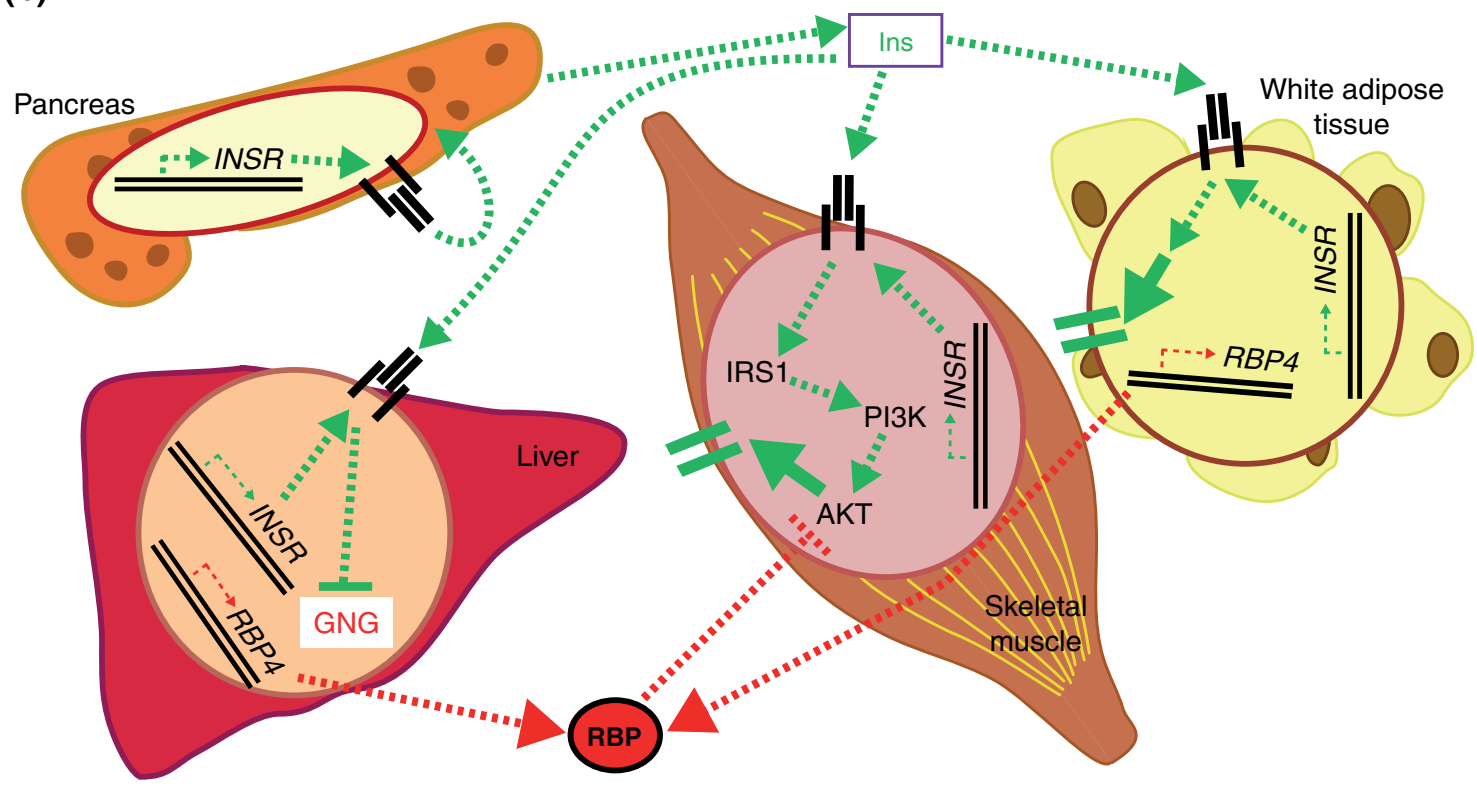

Figure 1

Model of the divergent consequences of HMGA1 deficiency. All actions that lower blood glucose are in green and influences that raise blood glucose are in red. Ins, insulin; GNG, gluconeogenesis. (a) In the normal physiological state, insulin action dominates, with RBP opposing insulin signaling in skeletal muscle. (b) In states of Hmga1 deficiency, both downregulation of insulin receptor expression, promoting insulin resistance, and RBP, promoting insulin sensitivity, are seen. 
expression and enhanced insulin sensitivity in $\mathrm{Hmgar}^{-/}$ mice. The first key evidence that this may be partly true comes from the demonstration that these mice do have severely reduced levels of $R b p 4$ mRNA and circulating RBP. Furthermore, the enhanced expression of Glut4 in muscle of the knockout mice is normalized by exogenous RBP, and the enhanced glucose-lowering effect of insulin in the knockout animals is markedly attenuated by the same treatment (Figure 1). In wild-type mice glucagon strongly stimulates expression of Hmga1 and then Rbp4, an effect absent in $\mathrm{Hmga1}^{-/}$mice. This suggests that Hmga1 is at least permissive for glucagon-induced stimulation of Rbp4 via a direct effect of Hmga1 on the Rbp4 promoter. Glucagon exerts cellular effects largely through the second messenger cAMP, and together with previous in vitro studies, this implicates cAMP as a proximal cellular regulator of both genes. However, exactly how RBP impairs insulin signaling is unclear: insulinstimulated phosphorylation of phosphatidylinositol 3-kinase (PI3K), a key proximal step in insulin's metabolic signaling, was said previously to be severely blunted in $\mathrm{Hmga1}^{-/}$animals [6], whereas phosphorylation of Akt, the next step in the pathway, is increased in these mice according to Chiefari et al. [1] (Figure 1). Moreover, $\mathrm{Rbp}^{-/}$ mice show enhanced insulin-induced phosphorylation of insulin receptor substrate 1 and activation of $\mathrm{PI}_{3} \mathrm{~K}$, also seemingly at odds with the new findings [9]. These considerations should motivate further signaling studies.

\section{'HMGA1opathy': a novel insulin resistance subphenotype?}

As with all interesting results, new lines of enquiry are suggested by the current findings. Mice with $10 \%$ of normal Insr expression would be expected to be severely insulin resistant, yet $\mathrm{Hmgar}^{-/}$mice are insulin hypersensitive. After administration of RBP they remain more insulin sensitive than wild-type controls, indicating that suppressed RBP in the knockouts accounts for only part of their insulin sensitization relative to mice with primary Insr defects. This raises the question of whether a wider perturbation of the secreted proteins related to insulin action could be at play. Germane to this is the finding that the generalized IR of humans with INSR mutations is biochemically distinct both from the IR of patients with other monogenic forms of severe IR and from 'common' IR, with adiponectin (from adipose tissue), insulin-like growth factor binding protein 1 and sex hormone binding globulin (IGFBP1 and SHBG, both from liver) and liver fat content all serving as good discriminators of the groups [7,10] (Table 1). It seems plausible that HMGA1-deficient humans or mice may show a third subphenotype of IR with a unique profile of insulin-responsive secreted factors, a possibility given credence by the finding that HMGA1 directly influences IGFBP1 expression, for example. Clarifying this may not only help to understand the complex metabolic derangement of HMGA1 deficiency, but may also define its unique biochemical 'fingerprint' that will aid ascertainment of further cases for study.

In the continuing struggle to understand fully the primary metabolic perturbation in T2DM, novel pathways and new connections are to be welcomed. Signaling uncertainties notwithstanding, the link between RBP and the unusual metabolic phenotype of the $\mathrm{Hmga1}^{-1}$ mice is intriguing, and the work that led to this finding illustrates the great power of combining biochemistry, genetics and physiology in mice and humans to yield novel mechanistic insights into the regulation of metabolism. The next installment of the story is awaited with interest.

\section{Acknowledgements}

$\mathrm{RS}$ is funded by the Wellcome Trust (Intermediate Clinical Fellowship 080952/Z/06/Z).

\section{References}

1. Chiefari E, Paonessa F, liritano S, Le Pera I, Palmieri D, Brunetti G, Lupo A, Colantuoni V, Foti D, Gulletta E, De Sarro G, Fusco A, Brunetti A: The cAMP-HMGA1-RBP4 system: a novel biochemical pathway for modulating glucose homeostasis. BMC Biol 2009, 7:24

2. Takata $Y$, Kobayashi M, Maegawa H, Watanabe N, Ishibashi $\mathrm{O}$, Shigeta $Y$, Fujinami A: A primary defect in insulin receptor in a young male patient with insulin resistance. Metab Clin Exp 1986, 35:950-955.

3. Imano E, Kadowaki H, Kadowaki T, Iwama N, Watarai T, Kawamori R, Kamada T, Taylor SI: Two patients with insulin resistance due to decreased levels of insulin-receptor mRNA. Diabetes 1991, 40:548-557.

4. Brunetti A, Manfioletti G, Chiefari E, Goldfine ID, Foti D: Transcriptional regulation of human insulin receptor gene by the high-mobility group protein HMGI(Y). FASEB J 2001, 15:492-500.

5. Reeves R, Beckerbauer L: HMGI/Y proteins: flexible regulators of transcription and chromatin structure. Biochim Biophys Acta 2001, 1519:13-29.

6. Foti D, Chiefari E, Fedele M, luliano R, Brunetti L, Paonessa F, Manfioletti G, Barbetti F, Brunetti A, Croce CM, Fusco A, Brunetti A: Lack of the architectural factor HMGA1 causes insulin resistance and diabetes in humans and mice. Nat Med 2005, 11:765-773.

7. Semple RK, Sleigh A, Murgatroyd PR, Adams CA, Bluck L, Jackson S, Vottero A, Kanabar D, Charlton-Menys V, Durrington P, Soos MA, Carpenter TA, Lomas DJ, Cochran EK, Gorden P, O'Rahilly S, Savage DB: Postreceptor insulin resistance contributes to human dyslipidemia and hepatic steatosis. J Clin Invest 2009, 119:315-322.

8. Kulkarni RN, Bruning JC, Winnay JN, Postic C, Magnuson MA, Kahn CR: Tissue-specific knockout of the insulin receptor in pancreatic beta cells creates an insulin secretory defect similar to that in type 2 diabetes. Cell 1999, 96:329-339.

9. Yang Q, Graham TE, Mody N, Preitner F, Peroni OD, Zabolotny JM, Kotani K, Quadro L, Kahn BB: Serum retinol binding protein 4 contributes to insulin resistance in obesity and type 2 diabetes. Nature 2005, 436:356-362.

10. Semple RK, Cochran EK, Soos MA, Burling KA, Savage DB, Gorden P, O'Rahilly S: Plasma adiponectin as a marker of insulin receptor dysfunction: clinical utility in severe insulin resistance. Diabetes Care 2008, 31:977-979.

Published: 27 July 2009

doi:10.1186/jbiol64

(C) 2009 BioMed Central Ltd 